\title{
Parameter optimization of fluted-roller meter using discrete element method
}

\author{
Yuxiang Huang*, Botao Wang, Yuxiang Yao, Shangpeng Ding, J unchang Zhang, Ruixiang Zhu \\ (College of Mechanical and Electric Engineering, Northwest A\&F University, Yangling 712100, Shaanxi, China)
}

\begin{abstract}
The most important performance indicator of a fertilizer metering mechanism is the evenness of fertilizer flow. In this study, a regression mathematical model between the key operating parameters of the fluted roller meter and the flow evenness was developed to simulate a fluted-roller meter for metering diammonium phosphate fertilizer using the discrete element method (DEM). The model was verified by bench test using the same equipment and parameters as the DEM model. Selected working parameters of the fluted-roller meter, including roll length $(L)$, roll rotational speed $(n)$, and flap angle $(\alpha)$ (for fertilizer discharge control), were optimized to maximize the flow evenness. Flow evenness was assessed by the coefficient of variation $(\mathrm{CV})$ of the discharging mass during the operation. The simulation and experiment results showed the similar trends, in terms of effects of machine parameters on the CV. The relative errors ranged from $0.2 \%$ to $34.6 \%$ with a mean of $10.5 \%$. This demonstrated that the DEM model was feasible to simulate the metering process of the fluted-roller meter. The machine parameters that significantly affected the values of $\mathrm{CV}$ in descending order were $\alpha, L$ and $n$. Both simulation and measurement results revealed that the optimal machine parameters, represented by the minimum value of $\mathrm{CV}$, were observed at $L=45 \mathrm{~mm}, n=55 \mathrm{r} / \mathrm{min}$ and $\alpha=22.5^{\circ}$. This combination of parameters returned CV values of $10.89 \%$ and $9.55 \%$ for simulations and measurements, respectively. The study provided useful information for guiding the design and selection of machine parameters for metering devices for fertilizer applications.
\end{abstract}

Keywords: discrete element method, fertilizer, flow evenness, fluted-roller meter, optimization DOI: $10.25165 /$ j.ijabe.20181106.3573

Citation: Huang Y X, Wang B T, Yao Y X, Ding S P, Zhang J C, Zhu R X. Parameter optimization of fluted-roller meter using discrete element method. Int J Agric \& Biol Eng, 2018; 11(6): 65-72.

\section{Introduction}

Fluted-roller meter is a volumetric flow metering mechanism, widely used for metering fertilizer and seeds ${ }^{[1,2]}$. The main machine parameters include roll diameter, length, and rotational speed. Other machine parameters are flute diameter and number of flutes. When using a fluted-roller meter, the flutes can be envisioned as individual pockets. Material fills each pocket and is carried into the discharge spout in a pocket by pocket outlet. Consequently, the material would be released pocket by pocket, which, by definition, is a discontinuous batch flow or a surging flow. Thus, the fertilizer flow is more or less uneven when using this type of metering mechanism ${ }^{[3]}$. However, this unevenness can be minimized through optimizing the machine parameters. In the case of field application, uneven flow of fertilizer will cause uneven fertilizer distribution in soil, which will affect the evenness of plant nutrient uptake after fertilizer application. Therefore,

Received date: 2017-06-20 Accepted date: 2018-07-18

Biographies: Botao Wang, Master candidate, research interests: agricultural mechanization engineering, Email: 1204614270@qq.com; Yuxiang Yao, Master candidate, research interests: agricultural mechanization engineering, Email: 928557834@qq.com; Shangpeng Ding, Master candidate, research interests: agricultural mechanization engineering, Email: 781472655@qq.com; Junchang Zhang, Associate Professor, research interests: agricultural mechanization equipment in arid areas, Email: zhangjunchang@126.com; Ruixiang Zhu, Professor, research interests: agricultural mechanization technology and equipment, Email: jdxy9223@nwsuaf.edu.cn.

*Corresponding author: Yuxiang Huang, $\mathrm{PhD}$, Associate Professor, research interests: agricultural Engineering, Northwest A\&F University, Yangling 712100, Shanxi, China. Tel: +86-29-87091111, Email: hyx@nwsuaf.edu.cn. minimizing the unevenness of fertilizer flow is important to plant growth.

In the past, to overcome the batch delivery drawback of fluted-roller meters, other types of metering devices were studied with the intention of obtaining continuous material flow. Helical fluted-roller meters, multi-flight screw meters, and belt-type meters are some examples ${ }^{[4-6]}$. These types of mechanisms meter material flowed in a more continuous way, compared to the conventional fluted-roller meters. However, traditional fluted-roller meters are simpler, less costly, and work well with free-flowing fertilizer. They can also deal with various shape and size of granules, such as fertilizer materials, and had a reasonably good performance for fertilizer applications ${ }^{[7]}$. This study focused on a fluted-roller meter for fertilizer application.

Measurement of fertilizer flow evenness is not an easy task. It involves capturing fast-moving particles. Special equipment is required to do this, and such equipment is not always available. In addition, measurements have a low accuracy and are time-consuming as well. Modeling would be a better approach to handle such applications. In recent years, the discrete element method (DEM) has been widely used to study motions of particles ${ }^{[8,9]}$. This numerical modeling method is able to capture the dynamics of individual particles, and has gained popularity for modeling flow of granular materials ${ }^{[10,12]}$.

One of the key model components in the DEM is to build model particles, especially irregular shaped particles, to represent the real particles to be simulated. The DEM basic particles are spherical and are simple and easy to use. However, using spheres to represent irregularly shaped particles can introduce errors in the model. Thus, the multi-sphere (MS) method has been proposed in the past for DEM simulations. The MS method dictates that a 
non-spherical particle of a material can be represented by connecting multiple spheres together ${ }^{[13,14]}$. Coetzee used 2-sphere, 4-sphere, and 8-sphere particles to represent crushed rock particles with three distinct shapes ${ }^{[15]}$. The simulations of hopper discharging of these model particles showed a variation in discharge rate with particle shapes, and each of those three model particles had a lower discharge rate than spherical model particles. This demonstrated that DEM model outputs are affected by the particle representations.

Little research has been done in the area of DEM modeling of fertilizer metering ${ }^{[8,10,13]}$. The most relevant work to this study was done by Hao et al. ${ }^{[16]}$ who simulated the metering process of a fluted-roller meter. In that simulation work, fertilizer material was modeled as spherical particles following a normal distribution, and the fertilizer discharging mass was examined under different machine parameters. Several other studies have been found on fertilizer spreading, these were reviewed as those applications also deal with particle movement. Coetzee and Lombard $^{[17]}$ modeled a centrifugal fertilizer spreader using the DEM. Fertilizer material was represented by an assembly of spherical particles. The work focused on examining effects of a deflector of the fertilizer spreader and model parameters on the fertilizer spreading patterns. Instead of using spherical particles, Van Liedekerke et al. ${ }^{[18]}$ used the MS method and built 2-sphere particles to represent fertilizer granules in a DEM model of a fertilizer spreader. These studies all concluded that the DEM was a valuable tool in development, testing, and calibration of granular handling equipment. However, most existing DEM-related studies dealt with spreading patterns of centrifugal fertilizer applicators. Processes of metering fertilizer have not been well documented. There is a lack of research on using the DEM for optimal design of machine parameters for maximum evenness of fertilizer delivery.

There are several factors which affect the flow evenness of metering process. In literature, a fluted-roller meter was tested to investigate the influence of flute diameter, roll length, rotating speedand roll rational speed on flow evenness of alfalfa seeds, the determined recommended ranges were $6-8 \mathrm{~mm}, 15-25 \mathrm{~mm}$, and 20-40 r/min, respectively ${ }^{[19]}$. A study conducted by Bansal et al. ${ }^{[20]}$ found that the operational parameters of a roller meter for metering seeds should be adjusted based on types of crops. Boydas and Turgut ${ }^{[21]}$ studiedthe studded feed rollers and observed that the flow evenness of seeds was significantly affected by the stud design and seed rate. Helical fluted-roller meters have been studied for metering ammonium sulfate and diammonium phosphate fertilizers ${ }^{[4]}$. The flute diameter, active roll length, helical angle, and roll rotational speed all significantly affected the flow evenness of fertilizer. The flow evenness also varied between the two different fertilizers, which had differing properties. Therefore, the performance of a metering device is fertilizer specific. Most existing studies were about metering of seeds, not fertilizers. The performance of fluted-roller meters should be studied under the most critical machine parameters.

The objectives of this study were to (1) develop a model to simulate the metering process of a fluted-roller meter using the EDEM software (DEM Solution Ltd, Edinburgh, UK); (2) use the model to predict the flow evenness under different machine parameters (roll length, roll rotational speed, and discharge flap angle); (3) determine a set of machine parameters which resulted in the optimal performance, in terms of flow evenness; (4) validate the model results using an experiment.

\section{Methodology}

\subsection{Model development}

A numerical model was developed to simulate the fertilizer metering process using the $\operatorname{EDEM}^{\mathbb{B}}$ (DEM-Solutions Corp., UK), a high quality DEM software package. The EDEM model had several components, including fertilizer particle presentations, modeling of a metering device, and flow monitoring logic. The following sections describe these components in greater detail.

\subsubsection{Metering device}

The metering device modeled was adopted from the standard metering device (JB/T 9783-2013 Standards). It consisted of a fertilizer hopper, a roll with seven flutes, a roll housing, and a flap (Figure 1). The hopper serves as a container which holds fertilizer material and feeds it to the flutes. During the metering process, the top flutes of the roll are filled with fertilizer material as the flows through the hopper to the roll. When the roll rotates about its shaft, fertilizer is metered down to the roll housing, passing through the flap, and is discharged through the spout. The flap angle could be adjusted to control the fertilizer flow rate. The flow rate can also be altered by adjusting the active length of the roll, defined as the length that is in contact with fertilizer. Changing the rotational speed of the roll will also change the fertilizer flow rate. The 3D CAD drawing of the metering device was developed in 1:1 scale. The main dimensions of the device are listed in Table 1. The 3D drawing was then converted to a ".STEP" file, so that it could be imported into the EDEM model to simulate the metering process.

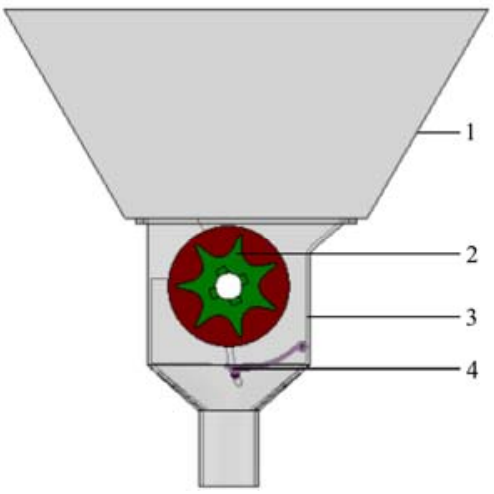

1. Fertilizer hopper 2. Fluted-roll 3. Roll housing 4. Discharging flap Figure 1 Drawing of the metering device

Table 1 Dimensions of the metering device

\begin{tabular}{lc}
\hline \multicolumn{1}{c}{ Dimension } & Value $/ \mathrm{mm}$ \\
\hline Roll diameter & 70 \\
Flute diameter & 26 \\
Hopper top & $320 \times 150$ \\
Hopper bottom & $160 \times 150$ \\
Height of hopper & 139 \\
Roll housing top & $146 \times 81$ \\
Roll housing bottom & $107 \times 65$ \\
Height of roll housing & 97.2 \\
\hline
\end{tabular}

\subsubsection{Shape presentations of fertilizer granules}

Diammonium phosphate (DP) fertilizer was used in this study, as it was a free-flow granular material which could be easily handled by fluted-roller meters. This fertilizer has also been commonly used for crop production. To fully represent the DP fertilizer granules in the model, the shapes and dimensions of a DP fertilizer granule were characterized by its length, width, and thickness. With these dimensions, the diameter of an equivalent 
sphere was determined using Equation (1). Then, the sphericity of the granule was calculated using Equation (2).

$$
\begin{gathered}
D=\sqrt[3]{L W T} \\
\Phi=\frac{D}{L}
\end{gathered}
$$

where, $L, W$ and $T$ are the measured length, width, and thickness of the fertilizer granule, $\mathrm{mm} ; D$ is the equivalent diameter of the granule, $\mathrm{mm} ; \Phi$ is the sphericity of the granule (dimensionless).

A representative sample of 50 granules was taken from commercial DP fertilizer. For each granule, the $L, W$, and $T$ were measured using a digital clipper (precision: $0.01 \mathrm{~mm}$ ). Then, the $D$ and $\Phi$ of the granule were determined from Equations (1) and (2). The measured values of $D$ from the 50 granules varied from $2.5 \mathrm{~mm}$ to $5.5 \mathrm{~mm}$. For simplicity, fertilizer granules were divided into three groups: small granules $(D<3 \mathrm{~mm})$, medium granules ( $3 \mathrm{~mm} \leq D \leq 4 \mathrm{~mm})$, and large granules $(D>4 \mathrm{~mm})$. The fraction (\%) of each group was calculated by the number of granules in that group divided by the total number of granules (50 in this case). Results showed that large granules were the most common fertilizer granules (Figure 2). The average values of measured $\Phi$ were $0.95,0.89$, and 0.84 for the small, medium, and large granule groups respectively. These $\Phi$ values demonstrated that the small granules were fairly round, the medium granules were less round, and the large granules were the least round. Given the fact that the least round granules accounted for the highest percentage $(70 \%)$ of the DP fertilizer, using spherical model particles to represent such a fertilizer would most likely introduce errors to model results.

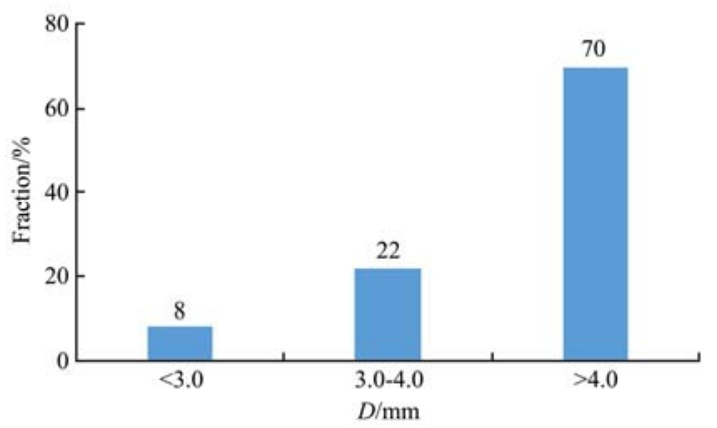

Figure 2 Fractions of different fertilizer size, in terms of equivalent diameter $D$

The next step was to obtain the shape of each DP fertilizer group using a 3D scanner (Makerbot) (Figure 3a). To obtain a granule scan, the granule was placed on the canner's rotational disc (Figure 3b). The scanner emitted two interlaced beams which were projected onto the surface of the granule. As the disc rotated, the laser beams scanned the entire surface of the granule, and registered the scan data every $2^{\circ}$ of disc rotation. This high resolution allowed for an accurate representation of the granule shape. After scanning was completed, the point cloud data was saved as ".STL" format, then imported into Geomagic Studio 2013 to remove noises and correct the defects of the data. In the end, a 3D "shell" was obtained, and it represented the surface of the scanned granule.

The aforementioned procedure was used to scan all three groups of DP fertilizer. The scanned shell of the small granule was close to spherical (Figure 4a), which was consistent with the measured sphericity of over 0.90 . The scanned shells of the medium and large granules were not as round, as shown in Figures $4 \mathrm{~b}$ and $4 \mathrm{c}$, which was also indicated by their sphericities of less than
0.90. Based on these results, spherical model particles were used to represent the DP granules in the small group (Figure $4 \mathrm{~d}$ ). The MS method was used to build clumps of the other two groups by populating the scanned shells with spherical balls ${ }^{[9]}$. The clump for the medium granules consisted of 15 balls and the ball diameter varied from $1 \mathrm{~mm}$ to $2 \mathrm{~mm}$ (Figure 4e), while the clump for the large granules was formed by 30 balls with diameters of 1-2 mm (Figure 4f).

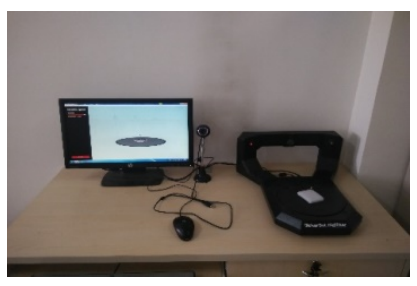

a. $3 \mathrm{D}$ scanner

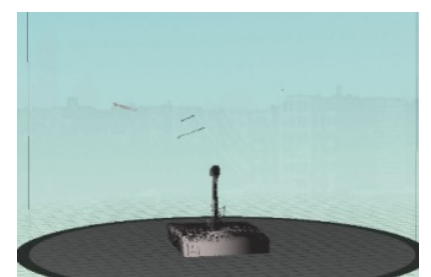

b. Rotational disc and fertilizer granule
Figure 3 Scanning of fertilizer granules

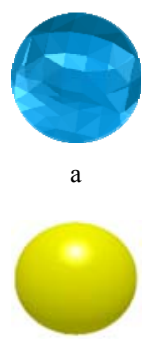

d

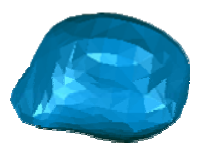

b

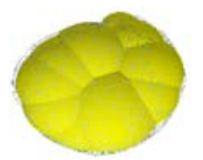

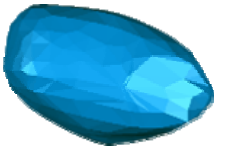

c

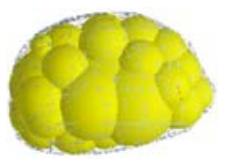

Figure 4 Mode fertilizer particles for (a)-(c) scanned shapes of small, medium, and large granules of DP fertilizer; (d)-(f) model

particle presentations of the small, medium and large granules

\subsubsection{Model parameters}

In simulations using EDEM, model parameters include parameters of materials and contacts between materials. In this case, the material parameters were density, Poisson's ratio, and shear modulus. The types of contacts included fertilizer to fertilizer (fertilizer-fertilizer), fertilizer-hopper, fertilizer-fluted roller meter contacts. The parameters of each contact were coefficient of restitution, static friction, and rolling friction. Researchers have conducted a lots of studies on model parameters, and the information on similar application to this study was available in the literatures ${ }^{[22-24]}$. Therefore, in this study, the parameters for the hopper and meter density, Poisson's ratio, and shear modulus were taken from the literature ${ }^{[23]}$. The shear modulus of the fertilizer was calculated using Equation (3).

$$
G=\frac{E}{2(1+v)}
$$

where, $G$ is the shear modulus, $\mathrm{Pa}$; $E$ is the modulus of elasticity, $\mathrm{Pa} ; v$ is Poisson's ratio. The values of $E$ and $v$ of the DP fertilizer were measured using a universal tester. The model parameters of materials are summarized in Table 2.

Table 2 Model parameters of materials

\begin{tabular}{lccc}
\hline \multirow{2}{*}{ Parameter } & Fertilizer & Hopper & Meter \\
\cline { 2 - 4 } & 1645 & 7810 & 1200 \\
\hline Density, $\rho / \mathrm{kg} \cdot \mathrm{m}^{-3}$ & 0.25 & 0.30 & 0.41 \\
Poison's ratio, $v$ & $72068,93648,122598^{*}$ & 206 & 113 \\
\hline Shear modulus, $G / \mathrm{kPa}$ &
\end{tabular}

Note: *The three values were for the small, medium and large granules respectively. 
Contact parameters describe behaviors of contacts between materials. Types of contact parameters depend on the contact model used. In this study, the Hertz-mindlin (no-slip) contact model was used for all contacts: contacts between fertilizer particles, fertilizer particles, and hopper, as well as fertilizer particles and meter. The contact parameters associated with Hertz-mindlin contact model were the coefficient of restitution (defined as the ratio of the impact velocity to the rebound velocity), static friction, and rolling friction. Static friction coefficients were measured using sliding tests, and rolling friction coefficients were measured using tests of the angle of repose ${ }^{[22]}$. To obtain the coefficient of restitution of the fertilizer, dropping tests were performed using a high-speed camera to capture the dropping and rebounding process of the fertilizer. The rebound height was obtained from the picture frames, and the coefficient of restitution was determined using the following equation:

$$
e=\sqrt{\frac{h}{H}}
$$

where, $e$ is coefficient of restitution; $H$ is the drop height of fertilizer granule; $h$ is the rebound height. Table 3 summarizes the contact parameters used for the model.

Table 3 Parameters of the contact model

\begin{tabular}{lccc}
\hline \multirow{2}{*}{ Parameter } & \multicolumn{3}{c}{ Contact } \\
\cline { 2 - 4 } & Particle-Particle & Particle-Hopper & Particle-Meter \\
\hline Restitution $(e)$ & 0.458 & 0.447 & 0.423 \\
Static friction $\left(\mu_{s}\right)$ & 0.300 & 0.301 & 0.220 \\
Rolling friction $\left(\mu_{r}\right)$ & 0.300 & 0.306 & 0.095 \\
\hline
\end{tabular}

\subsubsection{EDEM model of metering process}

The fertilizer particle models and metering device were integrated into an EDEM model to simulate the metering process of the DP fertilizer. In total, 10000 model fertilizer particles were generated in the hopper. Among the 10000 model particles, 800 were small granules represented by spheres, 2200 were medium granules and 7000 were large granules of multi-spherical presentations, based on the measured fraction ratio of the three DP granule groups of 8:22:70. To better visualize the fertilizer flow, green, red, and blue colors were used for the small, medium, and large granules respectively.

The input parameters listed in Tables 2 and 3 were specified in the EDEM model. The model was run by assigning a rotational speed to the fluted roll. A screenshot of the fertilizer flow process is shown in Figure 5. As the meter rotated, fertilizer particles were metered down and discharged through the flap. A receiving box was placed below the spout to collect the discharged fertilizer particles, which reduced the particle travel distances, and therefore, the computing time. The receiving box was placed far enough from the spout of the metering device, so it would not have any effect on the flow of fertilizer. The total simulation time was approximately $6 \mathrm{~s}$, with $2 \mathrm{~s}$ for generation of model fertilizer particles in the hopper, and $4 \mathrm{~s}$ for running the fluted-roller meter. A monitoring zone of $50 \mathrm{~mm}^{3}$ was created directly under the spout of the metering device, as shown in Figure 5. The monitoring zone could be used to observe any dynamic attributes of fertilizer particles. In this study, the observation was focused on the flow evenness of the discharged fertilizer over time. Therefore, only the mass of the fertilizer particles passing within the monitoring zone was monitored. The mass was recorded every $0.05 \mathrm{~s}$ during the time period of 2.35-6.0 s, giving a total of 73 readings. These readings were used to assess the performance of the metering device.

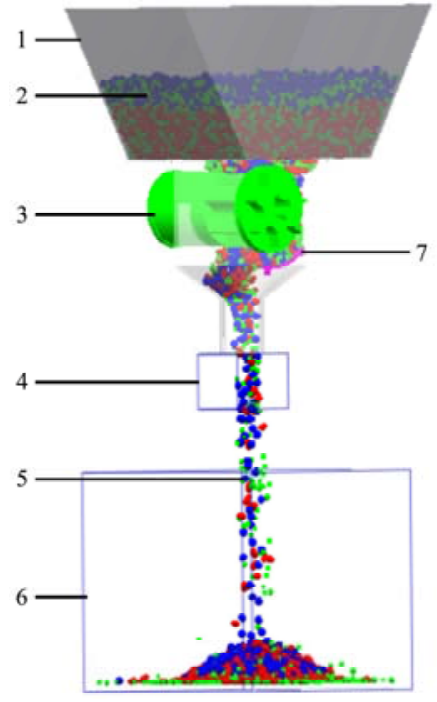

1. Hopper 2. Fertilizer particles 3. Fluted-roller meter 4. Monitoring zone 5. Flow of fertilizer 6 . Receiving box 7. Discharge flap

Figure 5 Screenshot of model metering process

\subsection{Optimization of machine parameters through simulation}

The EDEM model was run for various machine parameters to examine their effects on the machine performance. The objective was to find the set of machine parameters which resulted in optimal machine performance.

2.2.1 Objective function and constraints

Optimal machine performance of the metering device was considered to be the evenest flow. In other words, the optimization was to minimize the variation of the discharging mass during the operation. The coefficient of variation has been commonly used in literature to evaluate the performance of metering mechanisms ${ }^{[21]}$. Therefore, the objective function of the optimization was chosen as the coefficient of variation of the discharging. A minimum coefficient of variation indicated a maximum flow evenness, and consequently, optimal performance of the metering device. The coefficient of variation was determined using Equation (5).

$$
C V=\frac{\delta}{\sigma}
$$

where, $C V$ is coefficient of variation of samples of discharging mass; $\delta$ is sample mean, g; $\sigma$ is sample standard deviation, g. The sample mean was calculated using Equation (6), and the sample standard deviation was calculated using Equation (7).

$$
\begin{gathered}
\delta=\frac{\sum_{i=1}^{m} M_{i}}{m} \\
\sigma=\sqrt{\frac{\sum_{i=1}^{m}\left(M_{i}-\delta\right)}{m}}
\end{gathered}
$$

where, $i$ is sample index; $M_{i}$ is the mass of the $i^{\text {th }}$ sample, $g ; m$ is the number of samples.

The machine parameters selected to be optimized were the active roll length, roll rotational speed, and angle of the discharge flap, they are referred as roll length, roll speed, and flap angle, for simplicity. These have been considered to be the most influential machine parameters. The ranges commonly used were 20$70 \mathrm{r} / \mathrm{min}$ for roll speed, $10-60 \mathrm{~mm}$, for roll length, and the $0-30^{\circ}$ for flap angle ${ }^{[4]}$. Thus, the constraints for the optimization were established as the following equations:

$$
\begin{aligned}
10 \mathrm{~mm} & \leq L \leq 60 \mathrm{~mm} \\
20 \mathrm{r} / \mathrm{min} & \leq n \leq 70 \mathrm{r} / \mathrm{min} \\
0^{\circ} & \leq \alpha \leq 30^{\circ}
\end{aligned}
$$


where, $L$ is the roll length, $\mathrm{mm} ; n$ is the roll speed, $\mathrm{r} / \mathrm{mim} ; \alpha$ is the flap angle, $\left(^{\circ}\right)$.

The optimization process involved using the EDEM model to monitor the discharging mass $\left(M_{i}\right)$, within the monitoring zone, under the ranges of machine parameters listed in Equations (8)-(10). Then, using the $M_{i}$ values to determine the CV by Equations (5)-(7). The optimal machine parameters would be those which resulted in the lowest $\mathrm{CV}$.

\subsubsection{Design of optimization trials}

Before running the EDEM model to determine optimal machine performance, simulation trials were designed to have three factors (the three selected machine parameters) and five levels. Based on the constraints set in Equations (8)-(10), the $L$ was varied from $15 \mathrm{~mm}$ to $55 \mathrm{~mm}$ with $10 \mathrm{~mm}$ intervals, the $n$ was varied from $25 \mathrm{r} / \mathrm{min}$ to $65 \mathrm{r} / \mathrm{min}$ with $10 \mathrm{r} / \mathrm{min}$ intervals, and the $\alpha$ was varied from $0^{\circ}$ to $30^{\circ}$ with $7.5^{\circ}$ intervals. This gave a total of 20 trials based on the quadratic orthogonal rotation method (Table 4).

Table 4 Levels of machine factors: roll length $(L)$, roll speed $(n)$, and flap angle $(\alpha)$

\begin{tabular}{cccc}
\hline & \multicolumn{3}{c}{ Factor } \\
\cline { 2 - 4 } & $L / \mathrm{mm}$ & $n / \mathrm{r} \cdot \min ^{-1}$ & $\alpha /\left(^{\circ}\right)$ \\
\hline-2 & 15 & 25 & 0 \\
-1 & 25 & 35 & 7.5 \\
0 & 35 & 45 & 15 \\
1 & 45 & 55 & 22.5 \\
2 & 55 & 65 & 30 \\
\hline
\end{tabular}

\subsection{Experiment}

\subsubsection{Description of equipment and experimental design}

An experiment was conducted in the Agricultural Machinery Lab, Northwest A\&F University, China in October 2016 to collect data for model validations. In the experiment, the tested metering device had the same dimensions as the one used in the model. The meter device was mounted on a test stand that consisted of a belt, a motor, a drive system, and a speed control system (Figure 6). For the tests, a speed control system program was created to alter the motor speed, and therefore, the rotational speed of the fluted-roller meter. The roll length and flap angle were manually adjusted based on the scales on the metering device. The experimental design was designed using the same method, factors and levels as the simulations (Table 4), giving a total of 20 tests.

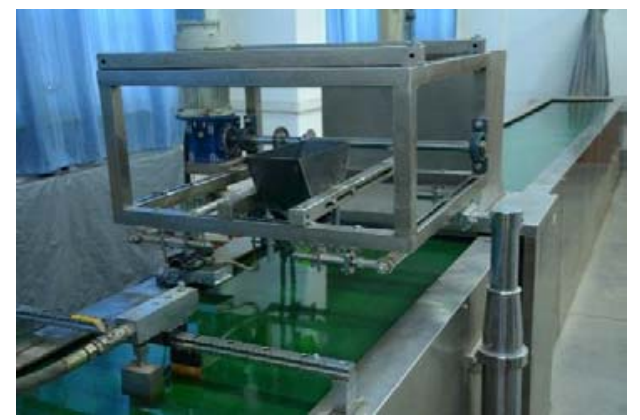

Figure 6 Test setup showing the meter device mounted on a test stand

\subsubsection{Measurements}

For testing, the belt speed of the test stand was set to $0.83 \mathrm{~m} / \mathrm{s}$ $(3 \mathrm{~km} / \mathrm{h})$. The hopper was filled with the DP fertilizer. As the fluted-roller meter rotated, fertilizer was metered down onto the belt, forming a fertilizer band on the belt (Figure 7). A section of $1 \mathrm{~m}$ on the fertilizer band was selected for measurements. This section represented the stable state of the metering process. Then, the band was divided into $30 \mathrm{~mm}$ segments. Fertilizer in each segment was collected and weighed to determine $M_{i}$. A similar measurement technique has been used to assess the uniformity of seed distribution by Malieki et al. ${ }^{[5]} \quad$ Using the $M_{i}$ values of those segments, the coefficients of variation $(\mathrm{CV})$ were calculated using Equations (5)-(7).

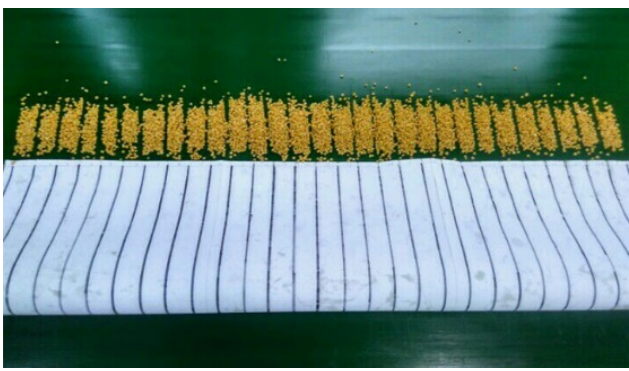

Figure 7 Divided fertilizer band on the belt of the test stand for measurements

\section{Results and discussion}

\subsection{Simulation results}

The simulated $\mathrm{CV}$ values for 20 different combinations of machine parameters were presented by response surfaces. A response surface of $\mathrm{CV}$ was formed by varying two machine parameters and keeping the other machine parameter constant. Figure $8 \mathrm{a}$ is the simulated surface response to a range of $L$ values $(15-55 \mathrm{~mm})$ and $n$ values $(25-65 \mathrm{r} / \mathrm{min})$ at a fixed level of $\alpha=15^{\circ}$. The "twisted" shape of the response surface demonstrated a complex combined effect of the two machine factors, and the nonlinear nature of the effect by $n$. Low CV values were located near the region with increased $L$ and $n$. However, high $C V$ values were not located at the opposite corner from the low region, but towards a reduced $n$ value. A higher surface slope was observed along the $n$ axis as compared with the $L$ axis, indicating that the effect of $n$ was dominant over that of $L$. As a result, the best performance was observed at the combination of $L=55 \mathrm{~mm}$ and $n=65 \mathrm{r} / \mathrm{min}$, where the $\mathrm{CV}$ was the lowest, indicating the highest flow evenness. Figure $8 \mathrm{~b}$ is the response surface of $\mathrm{CV}$ to various $\alpha$ values $\left(0^{\circ}-30^{\circ}\right)$ and $\mathrm{L}$ values $(15-55 \mathrm{~mm})$ at a fixed $n$ value (45 r/min). At $\alpha=0^{\circ}$, the $\mathrm{CV}$ was high, regardless of $L$. Along the $\alpha$ axis, the response surface curved down non-linearly. Larger $\alpha$ favored a lower $\mathrm{CV}$. The lowest $\mathrm{CV}$ region occurred in the region of increased $L$. The lowest $\mathrm{CV}$ was observed at $L=55 \mathrm{~mm}$ and $\alpha=30^{\circ}$. Figure $8 \mathrm{c}$ showed the response surface to various values of $n$ and $\alpha$ at a fixed $L(35 \mathrm{~mm})$. The response surface was curved in both directions. It descended non-linearly from the combination of the smallest $n$ and $\alpha$ to the combination of the largest $n$ and $\alpha$. The minimum CV was found at $n=65 \mathrm{r} / \mathrm{min}$ and $\alpha=30^{\circ}$.

\subsection{Optimal machine parameters}

Results from all 20 simulation trials are shown in Table 5. The values of simulated CV varied between $10.89 \%$ and $41.23 \%$, depending on the combination of machine parameters. Guler considered performances with CV values between $10 \%$ and $20 \%$ as "accepTable", while those below $10 \%$ as "good" $"[19]$. Given these criteria, among 20 combinations of machine parameters; one of them was good and 10 of them were acceptable. Any of these 11 combinations could be selected for the design of the fluted-roller meter to meter the DP fertilizer. However, for the best performance, the machine parameters should be $L=45 \mathrm{~mm}, n=$ $55 \mathrm{r} / \mathrm{min}$, and $\alpha=22.5^{\circ}$, where the simulated CV (10.89\%) was minimum. 

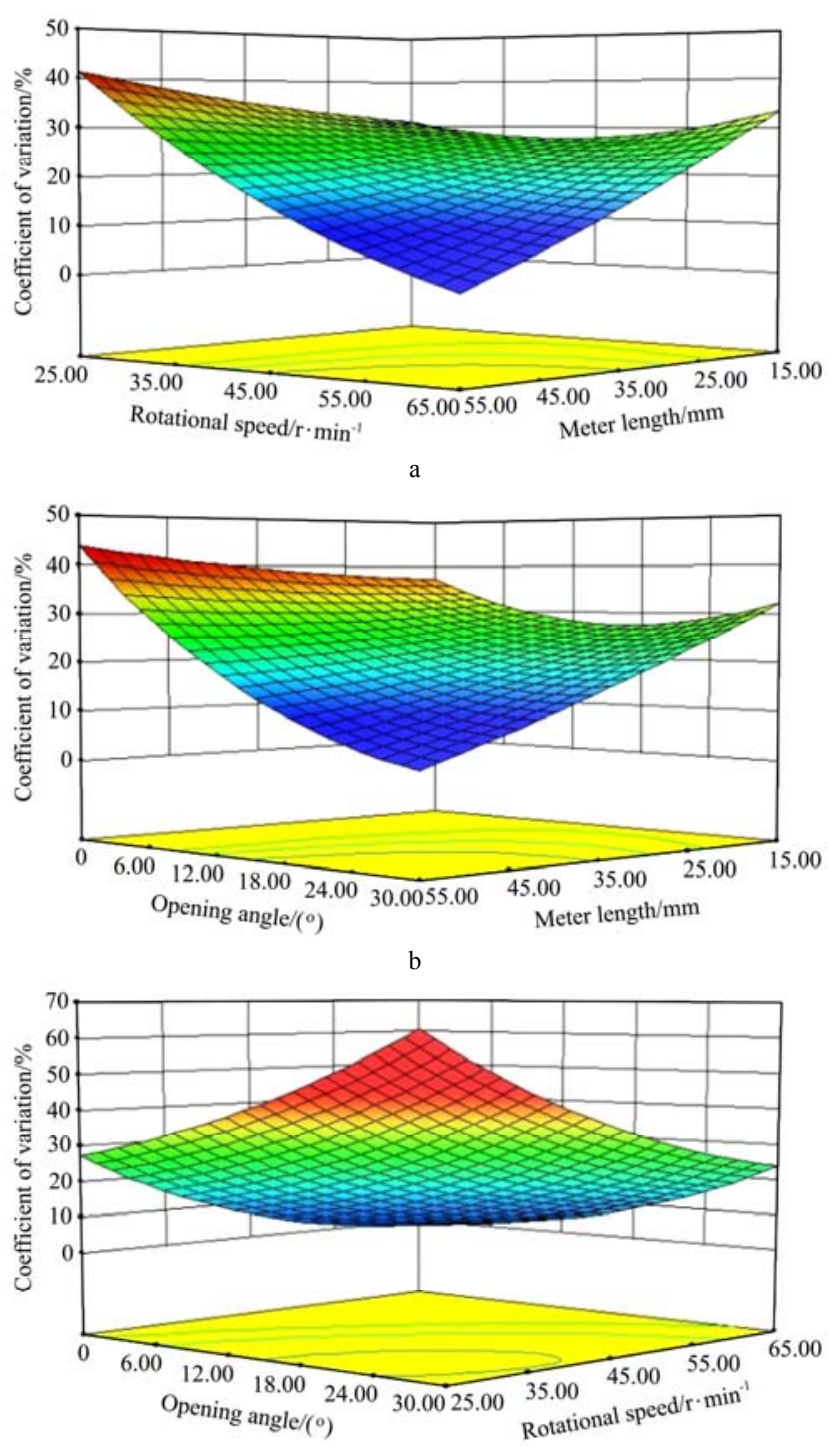

Figure 8 Response surfaces of the coefficients of variation $(\mathrm{CV})$ to machine parameters

\subsection{Model validations}

To validate the aforementioned simulation results, the simulated $\mathrm{CV}$ values were compared with those measured from the experiment (Table 5). A slightly narrower range of $\mathrm{CV}$ variations (9.55\%-38.97\%) was observed in the experiment. The agreement between simulations and measurements was assessed using relative errors (Table 5). Among the 20 combinations of machine parameters, the relative errors ranged from $0.2 \%$ to $34.6 \%$. The average relative error was $10.53 \%$. This low overall relative error indicated reasonably good model accuracy. Most importantly, the minimum measured $\mathrm{CV}, 9.55 \%$, value also occurred at the same combination of machine parameters as the simulation. This further proved the conclusion for the optimal combination of machine parameters. Similarly, the worst performance (i.e. the highest $\mathrm{CV}$ ) was at parameters $L=35 \mathrm{~mm}, n=45 \mathrm{r} / \mathrm{min}$, and $\alpha=0^{\circ}$, which was demonstrated by both the simulations and measurements.

Table 5 Comparisons in coefficient of variation (CV) between simulations and measurements under different roll length $(L)$, roll speed $(\mathrm{r} / \mathrm{min})$, and flap angle $(\alpha)$

\begin{tabular}{|c|c|c|c|c|c|c|}
\hline \multirow{2}{*}{$\begin{array}{c}\text { Trial } \\
\text { No }\end{array}$} & \multirow{2}{*}{$\stackrel{L}{L}$} & \multirow{2}{*}{$\begin{array}{c}N \\
/ \mathrm{r} \cdot \mathrm{min}^{-1}\end{array}$} & \multirow{2}{*}{$\begin{array}{c}A \\
/\left(^{\circ}\right)\end{array}$} & \multicolumn{3}{|c|}{$\mathrm{CV}$} \\
\hline & & & & Simulated $/ \%$ & Measured $/ \%$ & Relative error \\
\hline 1 & 25 & 35 & 7.5 & 28.2 & 24.0 & 17.6 \\
\hline 2 & 45 & 35 & 7.5 & 40.6 & 37.1 & 9.5 \\
\hline 3 & 25 & 55 & 7.5 & 28.2 & 31.2 & 9.6 \\
\hline 4 & 45 & 55 & 7.5 & 15.5 & 16.2 & 4.6 \\
\hline 5 & 25 & 35 & 22.5 & 25.9 & 25.0 & 3.5 \\
\hline 6 & 45 & 35 & 22.5 & 15.0 & 11.1 & 34.6 \\
\hline 7 & 25 & 55 & 22.5 & 18.3 & 18.8 & 2.8 \\
\hline 8 & 45 & 55 & 22.5 & 10.9 & 9.6 & 13.4 \\
\hline 9 & 15 & 45 & 15 & 29.8 & 28.8 & 3.3 \\
\hline 10 & 55 & 45 & 15 & 16.5 & 13.9 & 18.9 \\
\hline 11 & 35 & 25 & 15 & 36.6 & 35.2 & 4.0 \\
\hline 12 & 35 & 65 & 15 & 16.7 & 15.1 & 10.4 \\
\hline 13 & 35 & 45 & 0 & 41.2 & 39.0 & 5.8 \\
\hline 14 & 35 & 45 & 30 & 16.8 & 17.6 & 4.4 \\
\hline 15 & 35 & 45 & 15 & 18.9 & 17.2 & 10.0 \\
\hline 16 & 35 & 45 & 15 & 19.0 & 19.0 & 0.2 \\
\hline 17 & 35 & 45 & 15 & 18.2 & 24.5 & 25.7 \\
\hline 18 & 35 & 45 & 15 & 24.9 & 23.7 & 5.0 \\
\hline 19 & 35 & 45 & 15 & 18.6 & 21.9 & 15.0 \\
\hline 20 & 35 & 45 & 15 & 26.9 & 24.0 & 12.2 \\
\hline
\end{tabular}

Variance analysis was performed on the simulation and measurement data using the Design-Expert 8.0 software. The main effects of all three factors $(L, n$ and $\alpha)$ on $\mathrm{CV}$ were significant at $p<0.05$ (Table 6). The corresponding $F$ value ranked $L<n<\alpha$. This meant that the flap angle contributed the most to the variation of the fertilizer flow, whereas the roll length contributed the least. The variance analysis also showed that none of the interaction effects of these factors were significant. These statements were true for both the experiment and simulations.

Table 6 Variance analysis results of effects of roll length $(L)$, roll speed $(n)$, and flap angle $(\alpha)$

\begin{tabular}{|c|c|c|c|c|c|c|c|c|c|}
\hline \multirow{2}{*}{ Source } & \multirow{2}{*}{ DF } & \multicolumn{4}{|c|}{ Simulation } & \multicolumn{4}{|c|}{ Measurement } \\
\hline & & Mean square & $F$ value & $p$ value & Significance & Mean square & $F$ value & $p$ value & Significance \\
\hline Model & 9 & 0.01400 & 6.8 & 0.0030 & $* *$ & 0.01200 & 5.27 & 0.0079 & $* *$ \\
\hline$L$ & 1 & 0.01300 & 6.31 & 0.0308 & $*$ & 0.01900 & 8.19 & 0.0169 & $*$ \\
\hline$n$ & 1 & 0.03700 & 18.2 & 0.0016 & $* *$ & 0.02400 & 10.34 & 0.0092 & $* *$ \\
\hline$\alpha$ & 1 & 0.05200 & 25.81 & 0.0005 & $* *$ & 0.04700 & 20.41 & 0.0011 & $* *$ \\
\hline$L \alpha$ & 1 & 0.00402 & 1.99 & 0.1883 & & 0.00563 & 2.44 & 0.149 & \\
\hline$n \alpha$ & 1 & 0.00226 & 1.12 & 0.3148 & & 0.00043 & 0.19 & 0.6761 & \\
\hline$L^{2}$ & 1 & 0.00025 & 0.13 & 0.7298 & & 0.00025 & 0.11 & 0.7489 & \\
\hline$n^{2}$ & 1 & 0.00358 & 1.78 & 0.2123 & & 0.00104 & 0.45 & 0.5171 & \\
\hline$\alpha^{2}$ & 1 & 0.00808 & 4.01 & 0.0731 & & 0.00506 & 2.2 & 0.169 & \\
\hline Lack of Fit & 5 & 0.0026 & 1.82 & 0.2636 & & 0.003704 & 4.11 & 0.0736 & \\
\hline
\end{tabular}




\subsection{Discussion}

Values of CV varied over a large range in both the simulations and measurements. This could be explained by several facts. As mentioned before, the fertilizer flow from a fluted-roller meter is a discontinuous batch flow in nature. Thus, there is more or less flow surging to begin with when using a fluted meter. The extent of the surging is affected by the machine parameters, such as $L, n$, and $\alpha$. Changing any of these parameters would change the flow of fertilizer. There are limitations for increasing $L$ due to the limitation of physical space on a fertilizer applicator. There have been some mixed results in the literature regarding the effect of roll length. In a study by Su et al. ${ }^{[7]}$, the roll length appeared to have no effect on the CV. There are also limitations for increasing $n$ due to the limitation in target fertilizer rates. Thus, increasing $\alpha$ would be a practical means to achieve a uniform discharge rate by minimizing the $\mathrm{CV}$. Values of $\mathrm{CV}$ were most sensitive to $\alpha$, as demonstrated by the aforementioned response surfaces. The best performance of the fluted-roller meter was found to have a $\mathrm{CV}$ of $9.55 \%$. Values of $\mathrm{CV}$ around $8.4 \%$ were reported by $\mathrm{Su}$ et al. ${ }^{[7]}$ and they were considered to be good enough for a fluted-roller meter for fertilizer applications. Therefore, further $\mathrm{CV}$ improvement may not be necessary for the fluted-roller meter simulated in this study.

Fertilizer particles in the roll housing can be divided into three zones: forced moving zone, influenced zone, and steady zone. Fertilizer particles in the forced moving zone are those within the meter flutes, particles in this zone are forced to move by the rotating flutes and rotate together with the flutes. Particles in the influenced zone are those adjacent to the forced moving zone, and they are influenced by the rotating particles in the forced moving zone. As a result, particles in this zone were moved and discharged as well, due to particle-particle friction and interlocking between particles. However, the discharged fertilizer from the influenced zone is a more continuous flow. The steady zone was adjacent to the influenced zone, and particles in this zone do not move. In summary, fertilizer discharged from the meter come from two zones, and the discharge portion from each zone is controlled by the flap angle. At a smaller flap angle, most discharged fertilizer particles would be from the forced moving zone, where the surging nature of the fluted-roller meter was dominant. This explained the large $\mathrm{CV}$ at a smaller flap angle observed above. In contrast, at a larger flap angle, more particles were discharged from the influenced zone which was a continuously moving layer. This was why a large flap angle resulted in a smaller value of $\mathrm{CV}$.

\section{Conclusions}

A discrete element model was developed to simulate the metering process of a fluted-roller meter for fertilizer metering. The model results gave optimal machine parameters which resulted in the maximum evenness of fertilizer delivery. The model results were validated with experimental results. The following conclusions were drawn: 1) The model with irregular shaped fertilizer particles (clumps) was capable of simulating the dynamic flow of diammonium phosphate through a fluted-roller meter; 2) Effects of roll length, rotational speed, and flap angle all significantly affected the coefficient of variation (CV) of flow mass during the operation; 3) The best combination of machine parameters was determined to be a $45 \mathrm{~mm}$ roll length, rotating at $55 \mathrm{r} / \mathrm{min}$, with a flap angle of $22.5^{\circ}$; 4) Similar trends of CV responses to machine parameters were observed between simulation and experiment, which demonstrated that the discrete element model was feasible to optimize the machine parameters of the fluted-roller metering device.

Results from this study were for the given metering device and fertilizer type. Caution should be taken when applying the results for other applications. Also, some simplifications and assumptions were made when developing the model. Further research is required to verify the model results. The results can provide a theoretical basis for the discrete element simulation study of the fluted-roller meter and provide technical guidance for product design and practical operation.

\section{Acknowledgement}

This research was part of a project of Development of Technology and Equipment for Fertilizer Application based on 3S Technology, sponsored by the National Research and Development Program (2016YFD0200600, 2016YFD0200601).

\section{[References]}

[1] Zhang T, Liu F, Liu Y Q, Zhao M Q, Zhang S, Li N, et al. Discrete element simulation of outer groove wheel type fertilizer discharging device capacity analysis. Journal of Agricultural Mechanization Research, 2015; 9: 198-201. (in Chinese)

[2] Xia J, Xu Q, Wang Z, Zhou Y. Design of rice bud seed sowing and fertilizer machine. Transactions of the CSAM, 2010; 41(10): 44-47 (in Chinese)

[3] Lu X, Yang J, Chen S, Li Z. Design of straw returning and fertilization seeder's seeding device. Journal of Agricultural Mechanization Research, 2014; 289(38): 26395-26405. (in Chinese)

[4] Kara M, Bayhan A K, Özsert I, Yildirim Y. Performance of fluted roll metering devices in seed drills with ammonium sulphate and diammonium phosphate. Applied Engineering in Agriculture, 2010; 26(2): 197-201.

[5] Maleki M R, Jafari J F, Raufat M H, Mouazen A M, Baerdemaeker J. Evaluation of seed distribution uniformity of a multi-flight auger as a grain drill metering device. Biosystems Engineering, 2006; 94(4):535-543.

[6] Ess D R, Hawkins S E, Young J C, Christmas E P. Evaluation of the performance of a belt metering system for soybeans planted with a grain drill. Applied Engineering in Agriculture, 2005; 21(6):965-969.

[7] Su N, Xu T, Song L, Wang R, Wei Y. Variable rate fertilization system with adjustable active feed-roll length. Int J Agric \& Biol Eng, 2015; 8(4): 19-26.

[8] Abbaspour-Fard M H. Theoretical validation of a multi-sphere, discrete element model suitable for biomaterials handling simulation. Biosystems Engineering, 2004; 88(2): 153-161.

[9] Olieslagers R, Ramon H, Baerdemaeker J D. Calculation of fertilizer distribution patterns from a spinning disc spreader by means of a simulation model. Journal of Agricultural Engineering Research, 1996; 63(2): 137-152.

[10] Landry H, Laguë C, Roberge M. Discrete element representation of manure products. Computers \& Electronics in Agriculture, 2006; 51(1): 17-34.

[11] Chattha H S, Zaman Q U, Chang Y K, Read S, Schumann A W, Brewster G $\mathrm{R}$, et al. Variable rate spreader for real-time spot-application of granular fertilizer in wild blueberry. Computers and Electronics in Agriculture, 2014; 100(1): 70-78.

[12] Zeng Z, Chen Y, Zhang X. Modelling the interaction of a deep tillage tool with heterogeneous soil. Computers and Electronics in Agriculture, 2017; 143: $130-138$

[13] Mehatre R S, Dhomney S M, Sakhre D K. DEM simulation of coal particles for effective dispersion. Journal of Basic and Applied Engineering Research, 2014; 2(1): 43-45.

[14] Marigo M, Stitt E H. Discrete element method (DEM) for industrial applications: comments on calibration and validation for the modelling of cylindrical pellets. KONA Powder and Particle Journal, 2015; 32: 236-252.

[15] Coetzee C J. Calibration of the discrete element method and the effect of particle shape. Powder Technology,2016; 297: 50-70.

[16] Lv H, Yu J, Fu H. Simulation of the operation of a fertilizer spreader based 
on an outer groove wheel using a discrete element method. Mathematical and Computer Modelling, 2013; 58(3-4): 836-845.

[17] Coetzee C J, Lombard S G. Discrete element method modelling of a centrifugal fertiliser spreader. Biosystems Engineering, 2011; 109(4): 308-325.

[18] Liedekerke P V, Tijskens E, Ramon H. Discrete element simulations of the influence of fertiliser physical properties on the spread pattern from spinning disc spreaders. Biosystems Engineering, 2009; 102(4): 392-405.

[19] Guler I E. Effects of flute diameter fluted roll length, and speed on alfalfa seed flow. Applied Engineering in Agriculture, 2005; 21(1): 5-7.

[20] Bansal R K, Gharras O E, Hamilton J H. A roller-type positive-feed mechanism for seed metering. Journal of Agricultural Engineering Research,
1989; 43: 23-31.

[21] Boydas M G, Turgut N. Effect of vibration, roller design, and seed rates on the seed flow evenness of a studded feed roller. Applied Engineering in Agriculture, 2007; 23(4): 413-418.

[22] Huang Y, Hang C, Yuan M, Wang B, Zhu R. Discrete element simulation and experiment on disturbance behavior of subsoiling. Transaction of the CSAM, 2016; 47(7): 80-88. (in Chinese)

[23] Zhan Z, Li Y, Liang Z, Gong Z. DEM simulation and physical testing of rice seed impact against a grain loss sensor. Biosystems Engineering, 2013; 116(4): 410-419.

[24] Coetzee C J. Calibration of the discrete element method and the effect of particle shape. Powder Technology, 2016; 297: 50-70. 